\title{
EFEITO DO HIDROTREINAMENTO NA FORÇA MUSCULAR E CAPACIDADES FUNCIONAIS EM IDOSAS ATIVAS
}

\author{
J. C. G. Silva, A. M. Aboagarre Junior \\ Centro Universitario do Rio Grande do Norte Natal, Brasil.
}

OPEN ACCES

Correspondencia: Aboarrage junior Centro Universitário do Rio Grande do Norte (Brasil).

R. Prefeita Eliane Barros, 2000 Tirol, Natal - RN, 59014-54 ninosa@hotmail.com

Funciones de los autores: Todos los autores trabajaron equitativamente en la consecución del trabajo

Recibido: 8/06/2018 Aceptado: 19/11/2018 Publicado: 31/01/2019

Citación:

Silva, L. C. G., \& Aboagarre, A. M. (2019). Efeito do hidrotreinamento na força idosas ativas. RIAA. Revista de Investigación en Actividades Acuáticas, 2(3), 29-33. https://doi.org/10.21134/riaa.v3i5.1499

@creative

Creative Commons License Esta obra está bajo una licencia d Creative Commons ReconocimientoNoComercial-Compartir-lgua 4.0 Internacional
Contexto: $O$ envelhecimento é um processo natural nas alterações fisiológicas e determinam mudanças na estrutura do corpo humano, modificando suas funções, e essas modificações são caracterizadas por fatores genéticos, estilo de vida sedentária e ambientais

Objetivos: Avaliar o efeito de uma intervenção de Hidrotreinamento nas capacidades funcionais de idosas já treinadas no meio aquático.

Método: Foram recrutadas 60 idosas, todas já experientes no meio aquático para que não houvesse interferência na hora de dividir os grupos em Hidro treinamento $(n=30)$ com intervenção, e grupo controle $(n=30)$ com hidroginástica convencional, e na divisão dos grupos, foi de maneira aleatória, já que todas eram experientes em atividades aquáticas. As intervenções aconteceram durante 12 semanas, com 3 vezes por semanas. Os dados antropométricos e capacidades funcionais, foram realizados pré e pós intervenção Resultados: Houve diferença na força de preensão palmar hidro treinamento e o grupo controle com para mão dominante, e pós intervenção do hidro treinamento, e para o grupo controle para mão não-dominante. Para os testes funcionais, não houve diferença significativa nas variáveis de sentar e levantar, e levantar e caminhar.

Conclusões: $O$ presente estudo demostrou que a nossa hipótese de melhorar a força dos membros superiores e inferiores se confirmou, que uma intervenção de Hidro treinamento com exercícios específicos e bem orientados.

Palavras-chave: Preensão palmar, força isométrica, envelhecimento.

Título: Efecto del hidroentrenamiento en la fuerza muscular y capacidades funcionales en mayores activas

Introducción: El envejecimiento es un proceso natural en las alteraciones fisiológicas y determina cambios en la estructura del cuerpo humano, modificando sus funciones, y estas modificaciones se caracterizan por factores genéticos, estilo de vida sedentaria y ambiental.

Objetivos: Evaluar el efecto de una intervención de hidroentrenamiento en las capacidades funcionales de ancianas ya entrenadas en el medio acuático.

Método: Fueron reclutadas 60 ancianas, todas ya experimentadas en el medio acuático para que no hubiera interferencia a la hora de dividir los grupos en Hidrotrenamento $(n=30)$ con intervención, y grupo control $(n=30)$ con hidroginástica convencional, y en la división de los grupos, fue de manera aleatoria, ya que todas eran experimentadas en actividades acuáticas. Las intervenciones se produjo durante 12 semanas, con 3 veces por semanas. Los datos antropométricos y las capacidades funcionales se realizaron antes y después de la intervención.

Resultados: hubo diferencia en la fuerza de asimiento del palmar hidrotrenamento y el grupo control con para mano dominante, y post, y para el grupo control para mano no dominante. Para las pruebas funcionaesl, no hubo diferencia significativa en las variables de sentarse y levantar, y levantar y caminar.

Conclusiones: El presente estudio muestra que nuestra hipótesis de mejorar la fuerza de los miembros superiores e inferiores se ha confirmado, con una intervención de hidroentrenamiento con ejercicios específicos y bien orientados.

Palabras clave: Asimiento palmar, fuerza isométrica, envejecimiento.

Title: Effect of aquatic training in muscular force and functional capacities in elderly active

Introduction: Aging is a natural process in physiological changes and determines changes in the structure of the human body, modifying their functions, and these modifications are characterized by genetic factors, sedentary lifestyle and environmental. The Objectives: Evaluate the effect of a Hidrotreinamento intervention on the functional capacities of the elderly women trained in the aquatic environment.

Method: 60 elderly women were recruited, all of them already experienced in the aquatic environment so that there was no interference when dividing the groups in Hidrotreinamento $(n=30)$ with intervention, and control group $(n=30)$ with conventional hydrogymnastics, and in the division of groups, was in a random manner, since all were experienced in aquatic activities. Interventions occurred for 12 weeks, 3 times per week. Anthropometric data and functional abilities were performed before and after the intervention.

Results: There was a difference in the grip strength of the palmar hidrotreinamento and the control group with for dominant hand, and for the control group for non-dominant hand. For the functional tests, there was no significant difference in the sit and stand variables, and get up and walk.

Conclusions: The present study showed that our hypothesis to improve the strength of the upper and lower limbs was confirmed, that a Hidrotreinamento intervention with specific and well-oriented exercises.

Key words: Palmar grip, isometric force, aging. 


\section{Introdução}

O envelhecimento é um processo natural nas alterações fisiológicas determinam mudanças na estrutura do corpo humano, modificando suas funções, e essas modificações são caracterizadas por fatores genéticos, estilo de vida sedentária (Gómez-Cabello et al., 2012) e ambientais (Carbonell-Baeza et al., 2012; Wrycza; Missov; Baudisch, 2015) Com o envelhecimento os efeitos são acumulativos ao funcionamento orgânico, levando a um declínio das funções cognitivas, neuromusculares, e dificultando de maneira significativa as capacidades funcionais (Cunnane, Nugent, \& Roy, 2011).

As capacidades funcionais como força, resistência muscular e flexibilidade são umas das principais características que vão diminuindo ao passar do tempo, e as dificuldades nas atividades da vida diárias (AVDs) (Gómez-Cabello et al., 2012). Muitas dessas atividades são simples tarefas do cotidiano do idoso, tais como: levantar-se, ir ao banheiro e andar tornando-se cada vez mais difíceis. A redução da força e resistência compromete a mobilidade e equilíbrio, aumentando os ricos de quedas e de fraturas (Bird et al., 2009).

O risco de quedas pode ser atenuado com exercícios físicos como musculação, que ajuda na manutenção da massa muscular, melhorar no equilíbrio e força muscular, porém, esses danos podem gerar maior impacto nas articulações, estresse muscular, causando maior desistência por pessoas idosas (Gómez-Cabello et al., 2012). Algumas estratégias de exercício com baixo impacto, como exercício no meio aquático (Carbonell-Baeza et al., 2012), que geram menor impacto articulares, maior equilíbrio postural, força muscular, como isso, proporcionando maior confiança ao realizar os movimentos durante as aulas (Perigo Nascimento et al., 2016).

Porém, algumas intervenções realizadas no meio aquático geram melhoras em idosos obesos com dor lombar não localizada, mais não proporcionam tantas mudanças em determinadas capacidades funcionais e força muscular em idosas (Irandoust \& Taheri, 2015; Xiwei Zheng, Cong Bi, \& Brooks, 2015), com isso, nossa hipótese é que um treinamento periodizado melhore a força muscular e as capacidades funcionais em idosas já treinadas no meio aquático, portanto, o objetivo do estudo é, avaliar o efeito de uma intervenção de Hidro treinamento nas capacidades funcionais de idosas já treinadas no meio aquático.

\section{Método}

\section{Participantes}

Participaram do estudo, idosas com idade entre 60 a 75 (anos), altura de 150 a $165(\mathrm{~cm})$, massa corporal 50 a $80(\mathrm{~kg})$. Todas já realizavam hidroginástica no Instituto Federal do Rio Grande do Norte - IFRN. Todas foram convidadas a participar do estudo, assinando o Termo de Consentimento Livre e Esclarecido - TCLE. Foram incluídas, idosas sem queixas osteomuscular, doença cardiovascular, metabólica ou ortopédica, e que participavam de atividades aquáticas a mais de 6 meses, e como critério de exclusão, não ter assinado o TCLE.

Foram recrutadas 60 idosas, todas já experientes no meio aquático para que não houvesse interferência na hora de dividir os grupos em Hidro treinamento $(n=30)$ com intervenção, e grupo controle $(n=30)$ com hidroginástica convencional, e na divisão dos grupos, foi de maneira aleatória, já que todas eram experientes em atividades aquáticas.

\section{Procedimentos de avaliação e testes funcionais}

Na primeira visita, foram explicados cada tipo de teste, e possíveis desconforto de acordo com o tipo de teste. Todos os testes, foram realizados com 48 horas de intervalos, e sempre no mesmo horário anterior do teste anterior. Os testes funcionais seguiram os procedimentos de baterias de testes de Smith et al. (2018).

Dados antropométricos. As idosas realizam a medida da massa corporal em balança da balmak ${ }^{\circledR}$ com $100 \mathrm{~g}$ de erro, ficou em pé com os pés e posição confortável e braços unidos ao corpo, com roupas leves. Para a aferição da altura, se utilizou um estadiometro da Sanny ${ }^{\circledR}$ com erro de medida de $1 \mathrm{~cm}$. Durante a aferição, a idosa ficou em pé com a cabeça na posição de Frankfurd, os pés unidos e braços alinhados ao corpo. Durante a aferição, era informado que realizasse uma inspiração e segurasse durante 3 segundos, para melhor aferição da altura.

Preensão palmar. Para o teste de preensão palmar, foi utilizado o equipamento Dynamomenter SAEHAN ${ }^{\circledR}$, e foi realizado um ajuste prévio do equipamento das falanges dos dedos em cada idosa para melhor conforto. Durante a realização da força máxima, a idosa ficava sentada com as pernas e o braço flexionados a $90^{\circ}$ graus, sendo encorajada pelo avaliador a manter sempre o máximo de força durante os 5 segundos de teste, com intervalo de 10 segundos entre as tentativas, realizando 3 tentativas em cada membro, e para a troca de membro, descansava 5 minutos, e foram feitas as médias dos valores gerados da mão dominante e mão não-dominante (Bohannon, 1998).

\section{Diseño}

A intervenção teve duração de 12 (doze) semanas, 03 (três) vezes por semana, com treinos de 40 minutos de duração, totalizando 36 (trinta e seis) treinos, divididos em 3 etapas:

Primeira parte teve duração de 10 minutos de aquecimento com movimentos básicos para membros superiores (MMSS) e membros inferiores (MMII), elaborado com exercícios de fácil execução e com grande amplitude, sempre procurando uma área de superfície de contato pequena. À medida que repetia os movimentos com fluência, a área de contato iria aumentada, partindo para um aumento de intensidade.

Segunda parte, com duração aproximada de 20 minutos, foram desenvolvidos os seguintes exercícios, saltos verticais em cada bloco de treinamento. Salto Com Extensão do Quadril: inicia-se com uma flexão de quadril, joelhos e tornozelos, seguida por uma extensão rápida destas mesmas articulações, e aterrissando na mesma posição inicial conforme a ilustração abaixo. Salto Vertical Grupado: inicia-se com uma flexão de quadril, joelhos e tornozelos, seguida por uma extensão rápida destas mesmas articulações, na fase de voo a uma flexão de quadril e joelhos, aterrissando na mesma posição inicial conforme a ilustração abaixo. Salto Vertical com Abdução do Quadril: inicia-se com uma flexão de quadril, joelhos e tornozelos, seguida por uma extensão rápida destas mesmas articulações, na fase de voo promove-se uma forte abdução do quadril, aterrissando na mesma posição inicial conforme a ilustração abaixo. Salto Vertical com Adução do Quadril: inicia-se com os pés afastados aproximadamente na largura dos ombros, e com o quadril, joelhos e tornozelos, flexionados, seguida por uma extensão rápida destas mesmas articulações, mantendo os pés afastados, na fase de voo promove-se uma forte adução do quadril, aterrissando na mesma posição inicial (Aboarrage-Junior et al., 2018). Ainda na segunda parte foram executados com muito aceleração, número 5 na intensidade da tabela de Brenam (tabela 1), exercícios para membros superiores como: Adução e abdução dos ombros, flexão e extensão dos cotovelos, Adução e abdução horizontal dos ombros, todos eles realizado com a base fixa isto é sem a movimentação do membro inferiores, para dar maior estabilidade e foco no grupamento recrutado.

Todas as execuções foram monitoradas e corrigidas com o intuito melhorar os gestos motores, para poder ampliar a efetividade do treino. A intensidade foi subjetivada, seguindo a escala de percepção 
subjetiva de esforço exercido, sugerida por Brennan e Wilder (1990), de acordo com a tabela 01

Tabela 1. Escala de percepção subjetiva.

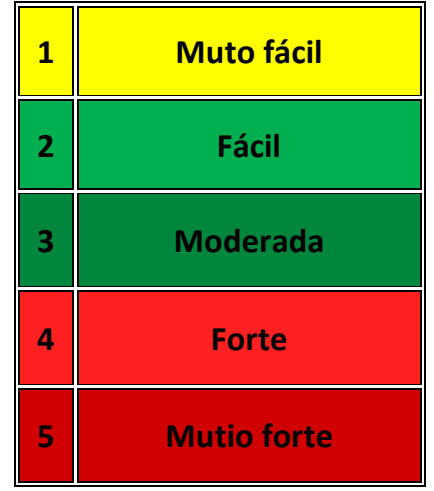

Finalizando com a terceira parte, foram realizados exercícios de alongamento e volta à calma, nos 10 minutos finais de cada encontro, para todos os grupos musculares, com ênfase nos membros inferiores.

Todos os treinos seguiram uma periodização pré-estabelecida, fo utilizado o protocolo de treino por (Aboarrage-Junior et al., 2018), consistem 4 séries de 30 segs. Estimulo seguindo 4 séries 30 segs. Recuperação (séries alternadas) com um tempo total de 4 minutos por bloco, e com 1 minuto de recuperação entre os blocos.

O Grupo Controle realizava a hidroginástica tradicional, com aquecimento de 10 minutos, exercícios como: corridas, chutes, polichinelos, twist, extensão do quadril, adução e abdução do quadril.

Na parte principal o professor repete os mesmos exercícios com mais intensidade trocando a cada um minuto durante 25 minutos e dependendo do dia, utilizava materiais como: macarrão e halter flutuante para membros superiores, e macarrão para ajudar na flutuação e fazer exercícios em suspensão para membros inferiores como abdução e adução e flexão e extensão do quadril simultaneamente. Segue as ilustrações abaixo alguns dos exercícios que foram usados no aquecimento e parte principal.

\section{Analises estatística}

Os dados foram discutidos após o teste de normalidade de Shapiro-wilk. As análises dos dados descritivos foram para idade, altura, massa corporal e IMC. As análises dos testes das capacidades funcionais estatísticas utilizando o teste $\mathrm{U}$ de Mann-Whitney, e para os dados da força de preensão palmar se utilizou o teste $t$ independente, utilizando um software estatístico especifico. A significância estatística foi estabelecida em $p<.05$, e para a geração dos gráficos, se utilizou o programa gerador de gráficos.

\section{Resultados}

No decorrer das 12 semanas, houveram 5 desistência por motivos não relatados, e 8 por faltar $25 \%$ das aulas/intervenção nos grupos, totalizando 47 participantes no final das 12 semanas. Os dados da idade, altura e composição corporal estão expressos na tabela 1. Os resultados dos testes funcionais estão nas figuras 1 e 2 .
Tabela 2. Características das participantes do estudo.

\begin{tabular}{|c|c|c|c|c|}
\hline \multirow[b]{2}{*}{$\begin{array}{l}\text { Caracterização } \\
\text { antropométrica }\end{array}$} & \multicolumn{2}{|c|}{$\begin{array}{c}\text { Hidro treinamento } \\
(n=25)\end{array}$} & $\begin{array}{c}\text { Grupo controle } \\
(n=22)\end{array}$ & \multirow[t]{2}{*}{$p$} \\
\hline & Pré & Pós & Pré & \\
\hline Idade (anos) & $60.10 \pm 2.92$ & $*$ & $66.40 \pm 4.22 *$ & $*$ \\
\hline Altura $(\mathrm{cm})$ & $1.56 \pm 0.05$ & $*$ & $1.52 \pm 0.10 *$ & $*$ \\
\hline \multirow[t]{2}{*}{ Massa Corporal (kg) } & \multicolumn{2}{|c|}{$68.66 \pm 10.05$} & $65.36 \pm 15.50$ & \multirow[t]{2}{*}{0.734} \\
\hline & \multicolumn{2}{|c|}{$68.90 \pm 10.54$} & $65.32 \pm 14.48$ & \\
\hline \multirow[t]{2}{*}{$\mathrm{IMC}\left(\mathrm{kg} / \mathrm{m}^{2}\right)$} & \multirow{2}{*}{\multicolumn{2}{|c|}{$28.43 \pm 4.453$}} & $28.54 \pm 8.15$ & \multirow[t]{2}{*}{0.58} \\
\hline & $26.54 \pm 4.84$ & & $28.56 \pm 4.38$ & \\
\hline
\end{tabular}

as 12 semanas de intervenção. IMC = índice de massa corporal.

Figura 1. Descrição do teste de levantar e caminhar pós intervenção de 12 semanas hidro treinamento $(p>0.254)$ e o grupo controle com $(p>$ 0.371).

Levantar e caminhar



Figura 2. Descrição do teste de levantar e caminhar pós intervenção de 12 semanas hidro treinamento $(p>0.254)$ e o grupo controle com $(p>$ 0.371).

Preensão palmar

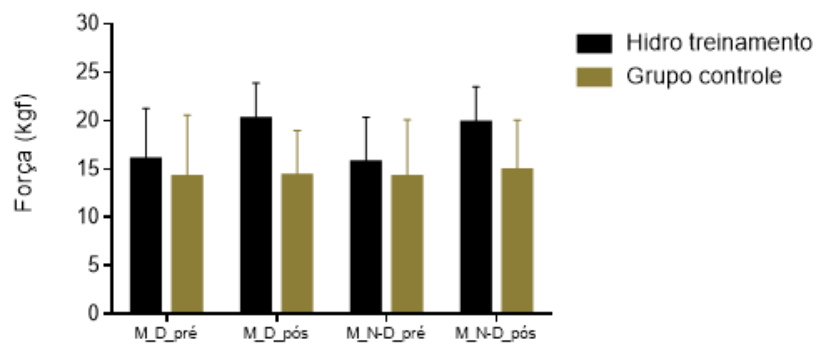

Discussão

Os nossos resultados mostraram que uma intervenção de 12 semanas de Hidro treinamento com exercícios específicos com padrão motor bem orientados, saltos variados, equipamentos de arrasto que auxiliam no aumento da resistência na água e distribuição de cargas em uma periodização planejada, podem melhorar as capacidades funcionais em idosas, e principalmente melhorando a força dos membros superiores quando comparado ao grupo que realizam hidroginástica convencional. Nossos resultados mostraram que o Hidro treinamento não foi capaz de diminuir o IMC e a massa corporal, quando observado ao grupo controle, isso, pode ser um indicador da falta de controle na ingesta calórica das participantes, pois isso não foi o foco da pesquisa, considerando que a intensidade do grupo hidro treinamento foi maior. Esses resultados também podem estar associados a familiarização com 
o meio aquática, já que todas as idosas praticavam hidroginástica. Como a composição corporal é um indicador de morbidade e pode influenciar na diminuição da funcionalidade do indivíduo na sua vida diária, como realizar uma caminhada, problemas ortopédicos em idosos, a hipótese do estudo era que esses parâmetros tivessem melhorias, pelo fato do maior volume de exercícios (Raffaelli et al., 2016; Schaun et al., 2018).

Figura 3. Descrição do teste de preensão palmar pós intervenção de 12 semanas hidro treinamento $(p<0.01)$ e o grupo controle com $(p>0.294)$ para M_D_(mão dominante), e pós intervenção do hidro treinamento ( $p$ $<0.01)$, e para o grupo controle $(p>0.110)$ para $M_{-} N-D_{-}$(mão nãodominante).

Preensão palmar

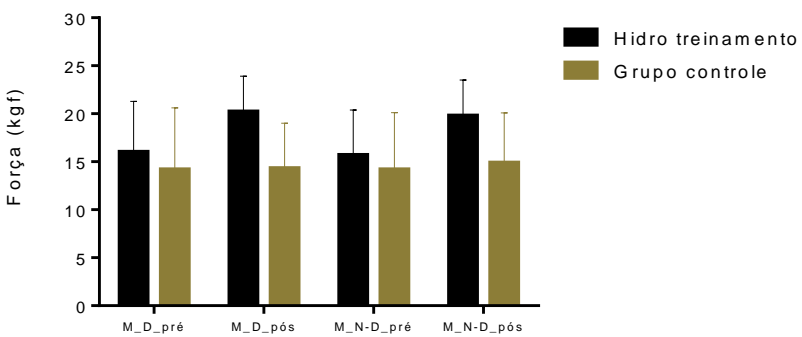

Em nosso estudo, o Hidrotreinamento não conseguiu melhorar de maneira significativa as capacidades funcionais, quando observado ao grupo controle. No entanto, as intervenções proporcionaram um menor tempo nas execuções da capacidade de realizar esse movimento, e possivelmente com melhor recrutamento muscular. Observando que a capacidade funcional de levantar e caminhar é caracterizada por realizar um movimento contínuo, que a uma relação das musculaturas agonista e antagonista, gerando um equilíbrio no sistema osteomuscular (Wilhem et al., 2014).

Para avaliar a força muscular global e a presença de sarcopenia usamos um dos métodos mais rápidos, simples e objetivos. Força de Preensão Manual é muito útil para avaliar a força muscular global de pessoas de meia-idade e idosos, sendo também um instrumento simples e bom preditor de prognóstico de saúde. recentemente, a força de preensão manual foi relatada como importante preditor do estado nutricional de indivíduos em situações de fragilidade, morbidade e mortalidade em indivíduos de meia-idade e idosos (Bohannon, 1998; Shiratori et al., 2014)

\section{Conclusões}

O presente estudo, demostrou que a nossa hipótese de melhorar a força dos membros superiores e inferiores se confirmou, que uma intervenção de Hidro treinamento com exercícios específicos e bem orientados, equipamentos de arrasto que auxiliam na resistência oferecida pela água, planejamento de exercícios físicos que utilizam membros inferiores e superiores ao mesmo momento pode auxiliar na manutenção da funcionalidade em idosas, e melhorando a força muscular de maneira geral.

\section{Contribuição e implicações práticas}

Acreditamos que este estudo pode contruibir muito para os profissionais que ministram aulas de hidroginástica, orientando-os na escolha dos exercicios e intensidades adequadas para causar uma adatação neuromuscular visando uma melhora na força e potência muscular, evitando e ou atenuando as quedas na sidosas, causadas pelo declínio das funções cognitivas, neuromusculares, e dificultando de maneira significativa as capacidades funcionáis (Cunnane et al., 2011).

\section{Agradecimentos}

Queremos agradecer a todos os profissionais e alunos que participararam do projeto e não mediram esforços para estar sempre presente nos treino e coletas. Ao Centro Universitario do Rio Grande do Norte, Centro de Atividades Aquáticas Hidroesporte e Instituto Federal do Rio Grande do Norte.

\section{Referencias}

Aboarrage, A. M., Teixeira, C. V. S., Dos Santos, R. N., Machado A. F., Evangelista, A. L., Rica, R. L.,... Bocalini, D. S. (2018). A high-intensity jump-based aquatic exercise program improves bone mineral density and functional fitness in postmenopausal women. Rejuvenation Research, 21(6), 535-540.

Bird, M. L., Hill, K., Ball, M., \& Williams, A. D. (2009). Effects of Resistance- and Flexibility- Exercise Interventions on Balance and Related Measures in Older Adults. Journal of Aging And Physical Activity, 17(4), 444-454.

Bohannon, R. (1998). Handgrip Dynamometry provides a valid indication of upper estremity strenth inpaiment in home care patients. Journal of Hand Therapy, 11, 258-260.

Carbonell-Baeza, A., Ruiz, J. R., Aparicio, V. A., Ortega, F. B., MunguíaIzquierdo, D., Alvarez-Gallardo, I. C... Delgado-Fernández, M. (2012). Land- and water-based exercise intervention in women with fibromyalgia: the al-Andalus physical activity randomised controlled trial. BMC Musculoskeletal Disorders, 13, 18.

Cunnane, S., Nugent, S., Roy, M., Courchesne-Loyer, A., Croteau, E., Tremblay, S., Castellano, A.,... Rapoport, S. I. (2011). Brain fuel metabolism, aging and Alzheimer's disease. Nutrition, 27(1), 3-20.

Gómez-Cabello, A. et al. (2012). Sitting time increases the overweight and obesity risk independently of walking time in elderly people from Spain. Maturitas, 73(4), 337-343.

Irandoust, K. \& Taheri, M. (2015). The effects of aquatic exercise on body composition and nonspecific low back pain in elderly males. Journal of Physical Therapy Science, 27(2), 433-435.

Perigo Nascimento, N. A. et al. (2016). Relation Among 25(Oh)D, Aquatic Exercises, and Multifunctional Fitness on Functional Performance of Elderly Women From the Community. Journal of Nutrition Health \& Aging, 20(4), 376-382.

Raffaelli, C. et al. (2016). Water-based training enhances both physical capacities and body composition in healthy young adult women. Sport Sciences for Health, 12(2), 195-207.

Schaun, G. Z. et al. (2018). Energy expenditure and EPOC between water-based high-intensity interval training and moderate-intensity continuous training sessions in healthy women. Journal of Sports Sciences, v. 00, n. 00, p. 1-8.

Shiratori, A. P. et al. (2014). Protocolos de avaliação da força de preensão manual em indivíduos com artrite reumatoide: uma revisão sistemática. Revista Brasileira de Reumatologia, 54(2), 140147.

Smith, L. et al. (2018). Depressive symptoms, handgrip strength, and weight status in US older adults. Journal of Affective Disorders, 238, 305-310.

Wilhelm, E. N., Rech, A., Minozzo, F., Botton, C. E., Radaelli, R., Teixeira B. C., \& Reischak-Oliveira, A. P. R. (2014). Concurrent strength and endurance training exercise sequence does not affect neuromuscular adaptations in older men. Experimental Gerontology, 60, 207-214.

Wrycza, T. F., Missov, T. I., \& Baudisch, A. (2015). Quantifying the shape of aging. PLOS ONE, 10(3), 1-18.

Xiwei Zheng, Cong B.I., Marissa Brooks \& D. S. H. (2015). HHS Public Access. Anal Chem., 25(4), 368-379. 